Article

\title{
Intrinsic Sensing Properties of Chrysotile Fiber Reinforced Piezoelectric Cement-Based Composites
}

\author{
Jianlin Luo ${ }^{1,2,3, *}$, Chunwei Zhang ${ }^{1,2, *}, \operatorname{Lu~Li}^{1,4}$, Baolin Wang ${ }^{3}$, Qiuyi $\mathrm{Li}^{1,2,5}$, \\ Kwok L. Chung ${ }^{1}$ (D) and Chao Liu ${ }^{1}$ \\ 1 School of Civil Engineering, Qingdao University of Technology, Qingdao 266033, China; \\ kuaile8lulu@126.com (L.L.); lqyxyn@163.com (Q.L.); klchung@qut.edu.cn (K.L.C.); alexlc@163.com (C.L.) \\ 2 Collaborative Innovation Center of Engineering Construction and Safety in Shandong Blue Economic Zone, \\ Qingdao University of Technology, Qingdao 266033, China \\ 3 Center for Infrastructure Engineering, School of Computing, Engineering and Mathematics, Western Sydney \\ University, Sydney, NSW 2751, Australia; b.wang@westernsydney.edu.au \\ 4 Department of Architecture Engineering, Dezhou Technology Vocational College Qingdao Campus, \\ Qingdao 266232, China \\ 5 School of Architecture Engineering, Qingdao Agricultural University, Qingdao 266109, China \\ * Correspondence: j.luo@westernsydney.edu.au (J.L.); zhangchunwei@qut.edu.cn (C.Z.); \\ Tel.: +86-532-8507-1693 (C.Z.)
}

Received: 8 August 2018; Accepted: 6 September 2018; Published: 7 September 2018

check for updates

\begin{abstract}
Lead-zirconate-titanate (PZT) nanoscale powder was first synthesized by the sol-gel method, then PZT and 0-3 type PZT/chrysotile fiber (CSF)/cement composite (PZTCC) wafers were fabricated after grind-mixing PZT powder with strontium carbonate and/or cement, ductile CSF in tandem with press-sintered process, respectively. The crystal structure (XRD), microstructure (SEM), piezoelectric properties after surface silver penetration, and polarization of the PZT and PZTCC wafer were investigated. Furthermore, self-sensing responses under either impulse or cyclic loading and micro-hardness toughness of PZTCC were also investigated. Results show that the incorporation of CSF and cement admixture weakens the perovskite crystalline peak of PZTCC; reduces the corresponding piezoelectric coefficient from $119.2 \mathrm{pC} / \mathrm{N}$ to $32.5 \mathrm{pC} / \mathrm{N}$; but effectively bridges the gap on the toughness between PZTCC and concrete since the corresponding microhardness with 202.7 MPa of PZTCC is close to that of concrete. A good linear and fast electrical response against either impulse or cyclic loading of the PZTCC is achieved with their respective sensitivity, linearity, and repeatability to $1.505 \mathrm{mV} / \mathrm{N}, 2.42 \%$, and $2.11 \%$. The sensing responses and toughness of PZTCC is encouraging as an intrinsic piezoelectric sensor for real-time health monitoring of ductile concrete structures.
\end{abstract}

Keywords: structural health monitoring; intrinsic sensor; piezoelectric cement-based composite; chrysotile fiber reinforcing; piezoelectric property

\section{Introduction}

The monitoring of the structural integrity of concrete structures under external static/dynamic loads ideally employ cement-embedded sensors with fast but highly-accurate responses [1-4]. As firstly documented by Chung et al. in 1993 [5], cement-based composites incorporating microscale or nanoscale conductive additives, such as steel fibers, carbon fibers [1,5-7], carbon blacks [8,9], nickel powders [10,11], semi-conducting nanoparticles [12], carbon nanotubes (CNTs) [1,13-19], carbon nanofibers [19,20], and graphene [21], have been intensively explored as intrinsic embedded sensors for detecting structural stress/strains via variations in their electrical parameters. However, the polarizations arising from the considerable existing pore electrolytes, in porous cement-based 
sensors, have significant detrimental effects on the stability and accuracy of the embedded sensors' electrical responses, even if these sensors are encapsulated [1,6,9]. Han et al. [22] showed that the piezoresistive sensitivity of $\mathrm{CNT} /$ cement composites declined with increasing water content. Kim et al. [23] improved sensitivity and stability of piezoresistive sensors for CNT/cement composites after excluding moisture.

Piezoelectric sensors, like $\mathrm{BaTiO}_{3}, \mathrm{KNbO}_{3}$, lead-zirconate-titanate (PZT), and polyvinylidene difluoride-based sensors are insensitive to humidity, and have fast responses and high-accuracies [24-29]. Amongst them, the PZT-type sensor has the highest piezoelectric actuating-sensing function and strain sensitivity. Being gain intensive, they have extensive applications in structural health monitoring (SHM) [26-29]. Song et al. [26] employed two low-cost PZT-based transducers as actuator-receivers of swept sine stress wave, and achieved a good relationship between the damage index, established via wavelet packet analysis, and the loading displacement of L-shaped concrete-filled variable thickness steel tube columns under cyclic loading. However, there are mismatch on the mechanical impedance responses obtained from a pure PZT-type sensor with the concrete when embedded in concrete structures. Furthermore, a pure PZT-type sensor has high density but low ductility, whose interfaces with concrete tend to fail in harsh environments under complicated loadings [27].

$\mathrm{Li}$ et al. first introduced a cement admixture into pure PZT to fabricate a piezoelectric cement-based composite (PCM) to compensate for these disadvantages in 2002 [30]. Dong et al. [31,32], Lan et al. [33], Huang et al. [34], Chaipanich et al. [35-37], Gong et al. [38,39], Pan et al. [40,41], Luo et al. [42], and Zhang et al. [43] reported that, compared with 1-3, 2-2 type PCM sensor [31,33], the $0-3$ type PCM sensor had more stable piezoelectric self-sensing behaviors with age and temperature treatment, superior compatibility and impedance-matching with concrete, where PZT granules were directly but randomly distributed in three dimension cement matrix without extra cutting and embedding processes [31,41]. Moreover, PZT powder in nanoscale can be facile pressed and sintered into PCM-based sensor or actuator for SHM with accurate stoichiometric composition, uniform morphology, and proper microstructure to ensure good interface compatibility and enhanced piezoelectric sensitivity [38]. However, the ductility of PCM is still relatively low especially where PCM sensors are embedded into long slim concrete structures comprised of fiber reinforced concretes, or ductile engineering cementitious composites [44].

The fabrication processes of the PZT nanoscale powder is vital to the performance of 0-3 type PCM sensor. Compared with the synthesis processes of mechanochemical [45,46], microwave radiation [47], hydrothermal [48], and coprecipitation [49], the sol-gels process is the most widely-used synthesis process for PZT nanoscale powder due to its resulting uniform composition, accurate stoichiometric ratio, and ultra-high purity under laboratory operation [50-52]. Mu et al. developed a modified sol-gel method with water and a little absolute ethanol as solvents and inorganic salts as raw materials to achieve a uniform single-phase PZT powders with an average size of $70 \mathrm{~nm}$ after annealing for $2.5 \mathrm{~h}$ at $700{ }^{\circ} \mathrm{C}[51]$.

In this study, PZT nanoscale powder was first synthesized by the sol-gel method [51]. A trace of strontium carbonate was introduced to diminish shrinkage and stabilize crystal phase during PZT wafer calcination process [53]. Furthermore, ductile chrysotile fiber (CSF) was added into the $\mathrm{PZT} /$ cement grind-mixture to fabricate the 0-3 type PZT/CSF/cement composite (PZTCC) wafer by press-sintering method [54]. Thirdly, the piezoelectric, toughness, and self-sensing properties of PZT and PZTCC wafers were characterized to develop a type of intrinsic and ductile piezoelectric sensor for SHM in complex structure with balanced piezoelectric sensitivity and toughness.

\section{Materials and Methods}

\subsection{Raw Materials}

Lead acetate $\left(\mathrm{PbC}_{4} \mathrm{H}_{6} \mathrm{O}_{4} \cdot 3 \mathrm{H}_{2} \mathrm{O}\right.$, mass fraction $\left.\geq 99.5 \%\right)$, zirconyl nitrate $\left(\mathrm{ZrO}\left(\mathrm{NO}_{3}\right)_{2}\right.$, mass fraction $\geq 99.5 \%)$, tetrabutyl titanate $\left(\mathrm{Ti}\left(\mathrm{OC}_{4} \mathrm{H}_{9}\right)_{4}\right.$, mass fraction $\left.\geq 98 \%\right)$ were all purchased from Shanghai 
Tongya Chemical Technology Development Co., Ltd. (Shanghai, China); Strontium carbonate $\left(\mathrm{SrCO}_{3}\right.$, mass fraction $\geq 99.5 \%$ ) was purchased from Sigma-Aldrich (Shanghai, China) Trade Co. Ltd. (Shanghai, China); Ethylene glycol $\left(\mathrm{HOCH}_{2} \mathrm{CH}_{2} \mathrm{OH}\right.$, mass fraction $\left.\geq 96 \%\right)$, glacial acetic acid $\left(\mathrm{CH}_{3} \mathrm{COOH}\right.$, mass fraction $\geq 99.5 \%)$, ammonia $\left(\mathrm{NH}_{3} \cdot \mathrm{H}_{2} \mathrm{O}\right.$, mass fraction $\left.=25-28 \%\right)$ were all obtained from Yantai Sanhe Reagent Co. Ltd. (Yantai, China); Dimethicone $\left(\left(\mathrm{C}_{2} \mathrm{H}_{6} \mathrm{OSi}\right)_{n}, \mathrm{AR}\right)$ and alumina $\left(\mathrm{Al}_{2} \mathrm{O}_{3}, \mathrm{AR}\right)$ were both obtained from Tianjin Yongda Reagent Co. Ltd. (Tianjin, China); Polyvinyl alcohol was obtained from Sinopharm Group Chemical Reagent Co., Ltd. (Shanghai, China); Conductive silver paste (DAD-87 type) was purchased from Shanghai Synthetic Resin Research Institute (Shanghai, China); Anhydrous ethanol $\left(\mathrm{C}_{2} \mathrm{H}_{5} \mathrm{OH}\right.$, mass fraction $\left.\geq 99.7 \%\right)$ was obtained from Shanghai Ebi Chemical Reagent Co., Ltd. (Shanghai, China); Distilled water was commercially available; Cement was purchased from Shanshui Cement Group Co., Ltd. (P.O. 52.5 type, Qingdao, China); CSF was obtained from Qinghai Chuangan Co. Ltd. (Mangya, China).

\subsection{Preparation of PZT Nanoscale Powders, PZT Wafer, and PZTCC Composite}

The sol-gel schematic method for synthesizing PZT nanoscale powders was demonstrated in Figure 1. Lead acetate, zirconyl nitrate, and tetrabutyl titanate were weighed to molar ratio for $r(\mathrm{~Pb}) / r(\mathrm{Zr}) / r(\mathrm{Ti})$ to 1:0.52:0.48. The resulting zirconyl nitrate-tetrabutyl titanate mixture was dissolved in $50 \mathrm{~mL}$ of water with a small amount ethylene glycol, and rigorously mixed together for $0.5 \mathrm{~h}$ at $60{ }^{\circ} \mathrm{C}$ in a collector thermostatic magnetic stirrer (DF-101S type, Zhengzhou Keda Machinery And Instrument Equipment Co., Ltd., Zhengzhou, China) filled with dimethicone to prepare the precursor. Then, the lead acetate was dissolved in ethylene glycol where $c\left(\mathrm{~Pb}^{2+}\right)$ was $15 \%$ [51], and slowly added to the abovementioned precursor. Glacial acetic acid and ammonia was used to adjust the $\mathrm{pH}$ value to around 4.5, and then reacted for another $2 \mathrm{~h}$ at $60{ }^{\circ} \mathrm{C}$ to fabricate the PZT sol. The PZT sol was dried at $100^{\circ} \mathrm{C}$, and residual organics were incinerated in a resistance furnace to form the pre-sintering PZT. The pre-sintered PZT was sintered for $2 \mathrm{~h}$ at $800{ }^{\circ} \mathrm{C}$ in a high-temperature furnace (XZK-3 type, Longkou Electric Furnace Factory, Longkou, China) [34]. The PZT nanoscale powder was obtained by grinding.

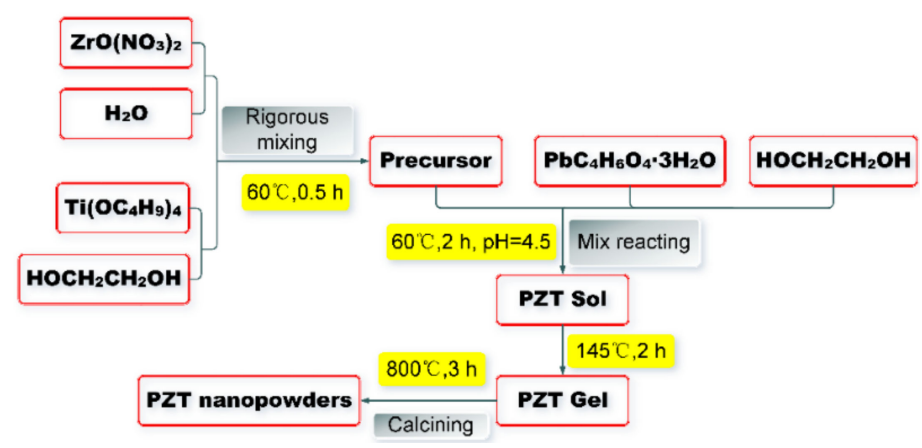

Figure 1. The sol-gel synthesis schematic procedure for lead-zirconate-titanate (PZT) nanoscale powders.

The PZT nanoscale powder, strontium carbonate ( $0.5 \%$ of PZT amount), for crystalline stabilization and deformation suppression, were glued together with polyvinyl alcohol and compressed into a wafer using a powder metallurgy tablet machine (HY-2H type, Tianjin Sinteron Powder Metallurgy Co., Ltd., Tianjin, China) for $2 \mathrm{~min}$ at $12 \mathrm{MPa}$. The PZT wafer had its glue removed by heating for $2 \mathrm{~h}$ at $300{ }^{\circ} \mathrm{C}$, and sintered for another $2 \mathrm{~h}$ at $800{ }^{\circ} \mathrm{C}$ with a surface covering alumina powder. The sintered PZT wafer was cleaned with anhydrous ethanol before a silver paste overlay was twice applied then heated for $3 \mathrm{~h}$ at $100{ }^{\circ} \mathrm{C}$. A $3000 \mathrm{kV} / \mathrm{cm}$ polarized voltage (DW-6000DC type, Tianjin Dongwen High Voltage Company, Tianjin, China) was applied for $30 \mathrm{~min}$ to the PZT wafer soaking in $120^{\circ} \mathrm{C}$ dimethicone solution [37].

The PZT nanoscale powder and strontium carbonate was ground together. CSF $(0.05 \%)$ and cement (25\% of PZT amount) was then added to the grinding process to create the PZT/CSF/cement 
(PZTCC) mixture, using anhydrous ethanol as a dispersing aid. An optical microscope (OP, XC4 type, Shanghai Precision Optoelectronics Co. Ltd., Shanghai, China) was used to observe the CSF in bundle and dispersed in anhydrous ethanol (Figure 2). The PZTCC mixture was compressed, glue removed, sintered, silver penetrated, and polarized as described in the PZT wafer preparation. It is worth noting that the PZTCC wafer, after sintering, was hydrated with a close fitting wet cotton wick, and oven-dried before silver penetration.

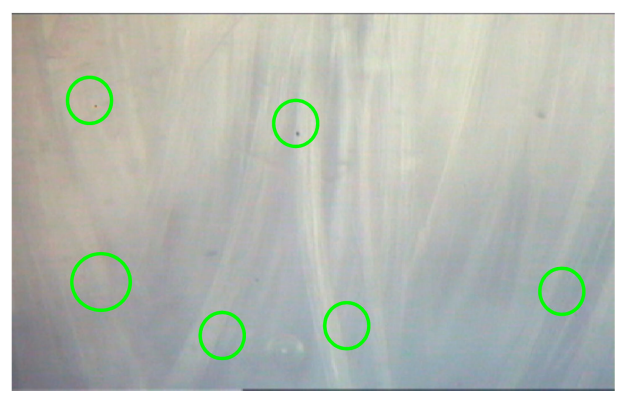

(a)

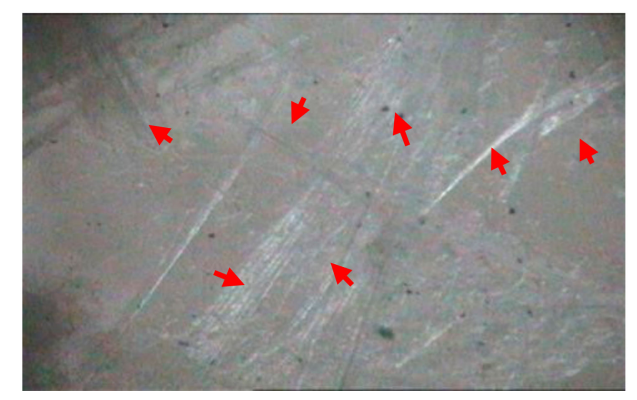

(b)

Figure 2. The optical morphologies of chrysotile fiber: (a) in bundle (green circles) $(\times 200)$; (b) dispersed state in ethanol (red arrows) after grinding for $30 \mathrm{~min}(\times 200)$.

\subsection{Characterization of PZT Nanoscale Powders, PZT Wafer, and PZTCC Composite}

X-ray powder diffraction (XRD, D8 Advance type, Bruker AXS Inc., Karlsruhe, Germany) and scanning electronic microscopy (SEM, S3500N type, Hitachi Co., Ltd., Tokyo, Japan) were used to characterize crystalline structures, particle sizes, and microstructures of PZT powder and PZTCC composites.

After surface polishing with different mesh sand papers, microhardness toughness measurement (HVS-1000 type, Laizhou Lyric Testing Equipment Co., Ltd., Laizhou, China) of the PZTCC composite was taken under a $1000 \mathrm{~g}$ load at $20 \mathrm{~s}$ compress duration. A quasi-static instrument (ZJ-6A type, Institute of Acoustics, CAS, Beijing, China) measured the $d_{33}$ coefficients of PZT and PZTCC wafer after polarization.

A dynamic data acquisition system (DASP-V10 type, Orient Institute of Noise \& Vibration, Beijing, China) measured the electricity responses versus hammer impulses at various loading levels $(100 \mathrm{~N}$, $200 \mathrm{~N}, 300 \mathrm{~N}, 400 \mathrm{~N}$ ) of PZTCC wafer with the top and bottom surfaces connected to a lead wire by conductive silver paste. DASP-V10 data acquisition system in tandem with a universal testing machine (E43 type, SANS-MTS, MTS Systems (China) Co., Ltd., Shenzhen, China) collected data from the applied loads and voltage responses of PZTCC wafer against time, and investigated the self-sensing property of PZTCC under cyclic loading with $0-100 \mathrm{~N}$ range at $2 \mathrm{~N} / \mathrm{s}$ head speed.

\section{Results and Disscussion}

\subsection{The Crystalline Structures of PZT and PZTCC Composite}

Figure 3 demonstrates the crystalline structures of PZT and PZTCC composite.

Figure 3 shows characteristic peaks for PZT $\left(2 \theta=21.3^{\circ}, 31.0^{\circ}, 38.4^{\circ}, 45.2^{\circ}, 49.5^{\circ}\right.$, and $\left.54.8^{\circ}\right)$, which represent the (100), (110), (111), (200), (201), and (211) patterns of PZT sintering for $2 \mathrm{~h}$ at $800{ }^{\circ} \mathrm{C}$. Trace strontium carbonate shows no effect on the crystalline structure of PZT and the main peak (110) of $\mathrm{PZT}$ is $\mathrm{Pb}\left(\mathrm{Zr}_{0.52} \mathrm{Ti}_{0.48}\right) \mathrm{O}_{3}$ crystal phase, which is the classic $\mathrm{ABO}_{3}$ type perovskite [53]. Although the addition of cement and CSF in PZTCC is insignificant on the basic crystal structures of PZT, several miscellaneous peaks appear, and their corresponding main peak (110) is also reduced, contributing to an inferior perovskite structure due to cement and CSF introduction (Figure 3). 


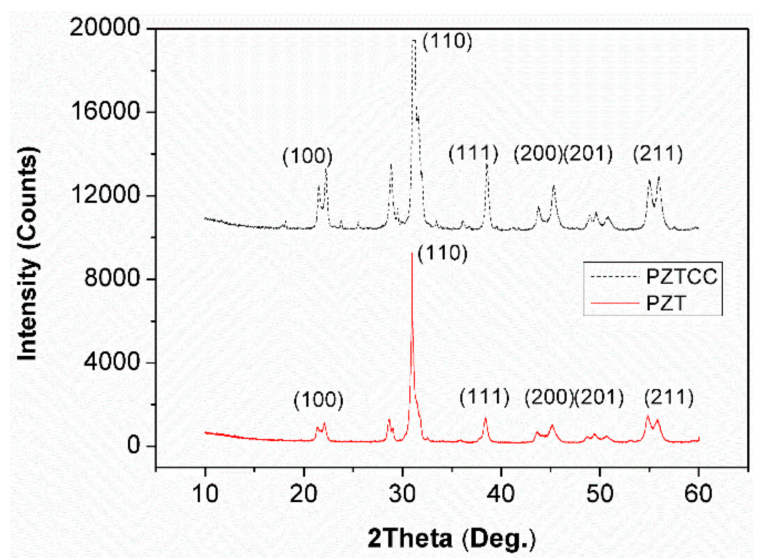

Figure 3. XRD crystallinity of PZT and PZTCC (PZT/CSF/cement composite).

\subsection{Microstructure and Piezoelectric Coefficient of PZT and PZTCC Composite}

Fine and even cubic crystallizations of PZT are observed in Figure 4a, the crystal sizes are narrowly distributed in the 900-1100 nm range [38]; the corresponding $d_{33}$ coefficient is up to $119.2 \mathrm{pC} / \mathrm{N}$. In Figure $4 \mathrm{~b}, \mathrm{CSF}$ and cement without any piezoelectric effect are dispersed within the PZT perovskite crystals, and perovskite PZT crystals are comparatively larger than those in the PZT wafer, which reduces the corresponding piezoelectric macro-performance of the PZTCC wafer, whose $\mathrm{d}_{33}$ coefficient is only $32.5 \mathrm{pC} / \mathrm{N}$.

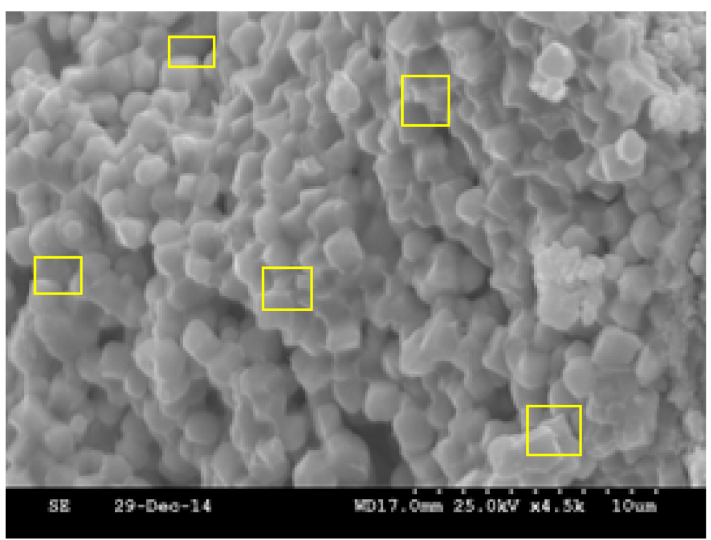

(a)

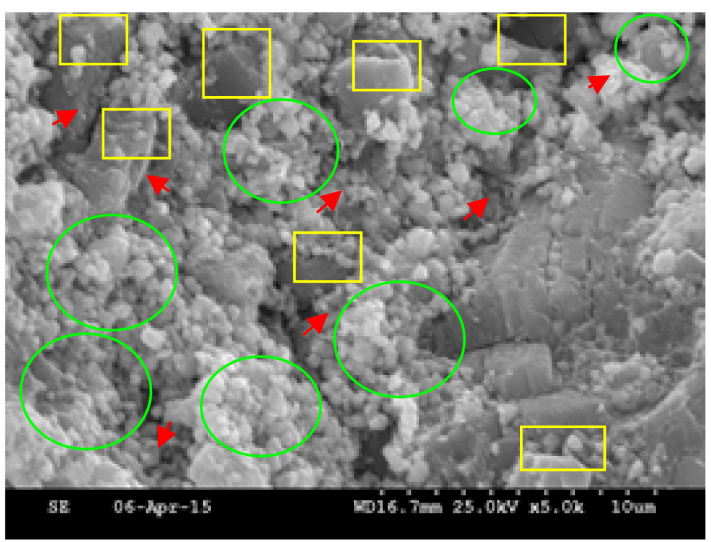

(b)

Figure 4. SEM images of PZT and PZTCC composite: (a) PZT (yellow rectangle, PZT); (b) PZTCC (yellow rectangle, PZT; green ellipse, cement particle; red arrow, CSF).

\subsection{Microhardness Toughness Properties of PZTCC Composite}

Figure 5 show surface images of the surface-polished PZTCC composite at macro-scale and as observed in OP. The interface microhardness of the PZTCC composite is $202.7 \pm 4.9 \mathrm{MPa}$ and the interface width is $20 \pm 0.3 \mu \mathrm{m}$. Compact interface connections between PZT nanoscale crystals among the cement matrix are achieved, also verified in Figure $4 \mathrm{~b}$. The resultant interface hardnesses are similar to common concrete [55], and suited to applications as embedded intrinsic sensors in concrete structures. 


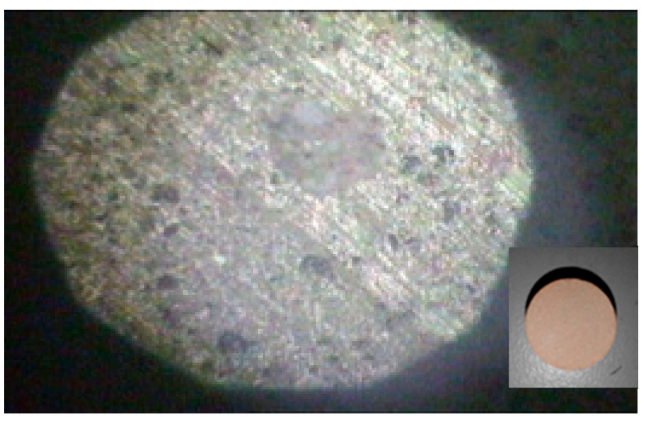

(a)

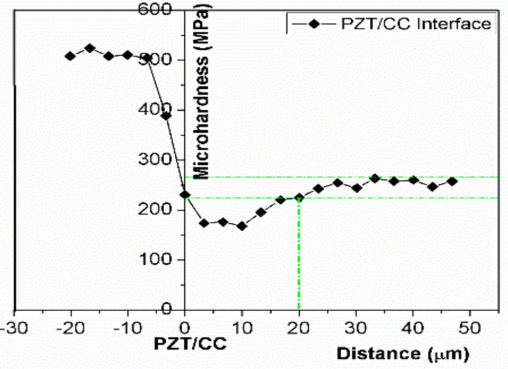

(b)

Figure 5. (a) An optical microscope image of the surface-polished PZTCC composite $(\times 400)$ and (the insert bottom-right picture of the PZTCC composite at macro-scale); (b) Micro-hardness distributions at the PZT/CC interface.

\subsection{Piezoelectric Sensing Properties of PZTCC Wafer}

The electrical voltage time-history of the PZTCC wafer under various impulse levels and cyclic loads are recorded and illustrated in Figures 6 and 7, respectively.

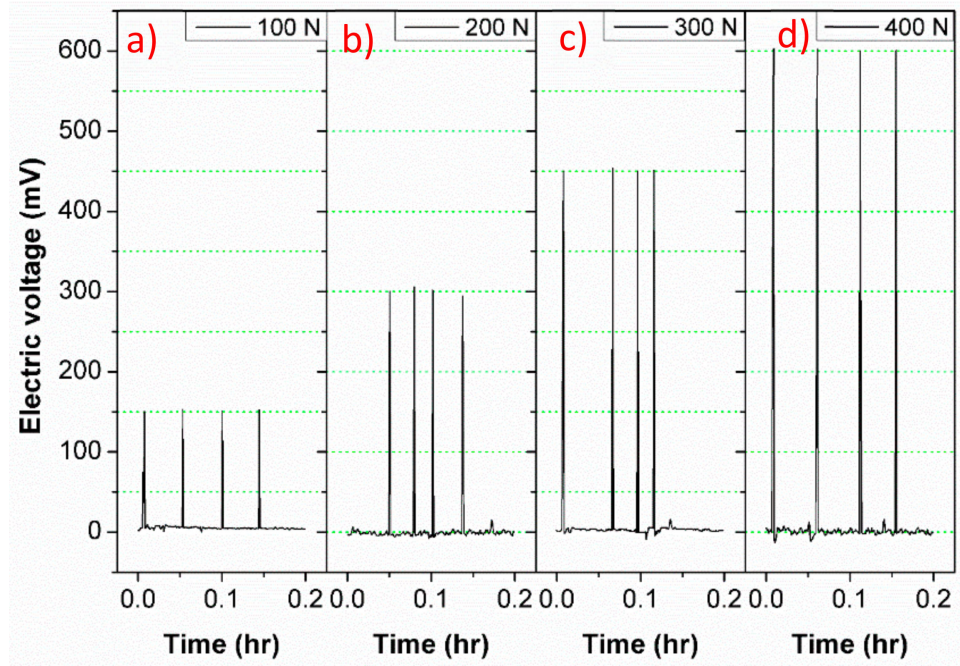

Figure 6. Electric voltage versus time of the PZTCC wafer under various impulse levels: (a) $100 \mathrm{~N}$; (b) $200 \mathrm{~N}$; (c) $300 \mathrm{~N}$; (d) $400 \mathrm{~N}$.

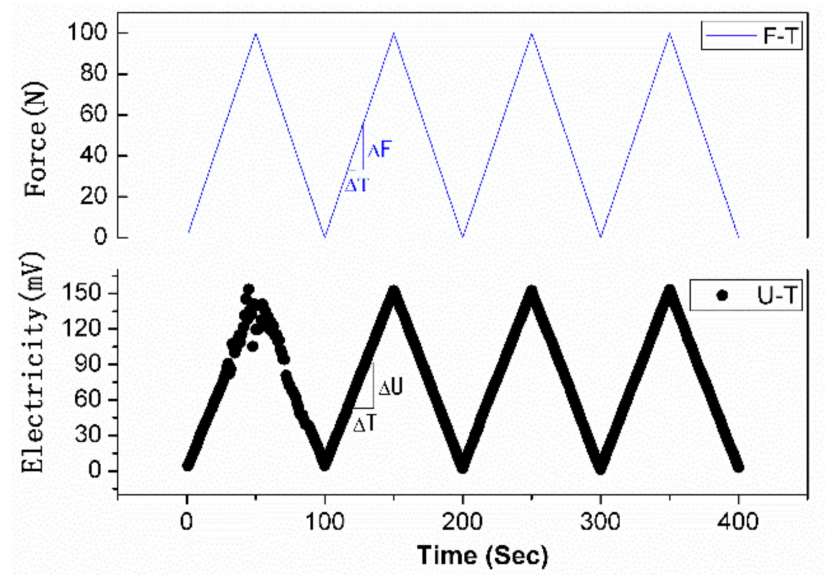

Figure 7. Electricity history of PZTCC wafer under cyclic loading. 
In Figure 6, the electricity output almost retains $150 \mathrm{mV}, 300 \mathrm{mV}, 450 \mathrm{mV}$, and $600 \mathrm{mV}$ against their corresponding $100 \mathrm{~N}, 200 \mathrm{~N}, 300 \mathrm{~N}, 400 \mathrm{~N}$ impulses, over four repeat tests. It shows both a good linearity and a rapidity of the electrical response to the impluse level, although the $d_{33}$ coefficient is relatively lower than that of common PZT wafer [35]. Figure 7 shows that the electrical voltages vary linearly with the applied cyclic force, although there is an initial fluctuations at the initial loading cycle.

The linearity $(e)$, sensitivity $(k)$ and repeatability $\left(e_{\mathrm{f}}\right)$ of the sensor paramters of the PZTCC wafer under cyclic loading can be calculated by the following formulas $[1,8]$,

$$
\begin{gathered}
e= \pm \Delta_{\max } / \mathrm{U}_{\mathrm{F} . S} \times 100 \% \\
k=\Delta \mathrm{U} / \Delta \mathrm{F} \\
e_{\mathrm{f}}= \pm \Delta_{\max }^{\prime} / 2 \mathrm{U}_{\mathrm{F} . \mathrm{S}} \times 100 \%
\end{gathered}
$$

The $\Delta_{\max }, \mathrm{U}_{\mathrm{F} . S}, \Delta \mathrm{U}, \Delta \mathrm{F}$, and $\Delta_{\text {max }}^{\prime}$ represents the maximum deviation between the electricity (U)- force (F) curve and the linear fitting line, full-scale output of $U$, change in $U$, change in $F$, and maximum deviation in forward and reverse stroke of U-F curve, respectively.

Table 1 shows the calculated $e, k$, and $e_{\mathrm{f}}$ parameters with their corresponding means, derived after excluding the fluctuations of cycle 1 .

Table 1. The linearity $(e)$, sensitivity $(k)$, and repeatability $\left(e_{\mathrm{f}}\right)$ of the PZTCC under cyclic loading.

\begin{tabular}{cccccc}
\hline Parameter & Cycle 1 & Cycle 2 & Cycle 3 & Cycle 4 & Mean \\
\hline \multirow{2}{*}{$e$} & $25.94 \%$ & $3.04 \%$ & $2.86 \%$ & $2.41 \%$ & \multirow{2}{*}{$2.42 \%$} \\
& $16.08 \%$ & $2.18 \%$ & $2.01 \%$ & $2.02 \%$ & \\
\multirow{2}{*}{$k$} & 1.1751 & 1.5080 & 1.4973 & 1.5132 & \multirow{2}{*}{$1.505 \mathrm{mV} / \mathrm{N}$} \\
& 1.3844 & 1.5064 & 1.5049 & 1.5003 & \\
\hline$e_{\mathrm{f}}$ & $2.4 \%$ & $2.4 \%$ & $1.5 \%$ & $2.42 \%$ & $2.11 \%$ \\
\hline
\end{tabular}

It can be determined from Figure 7 and Table 1 that, the sensitivity $k$ is $1.505 \mathrm{~V} / \mathrm{kN}$, which suits most civil engineering applications [1]. Further, both $e$ and $e_{\mathrm{f}}$ parameters are well below $5 \%$, and their correponding means are only $2.42 \%, 2.11 \%$, respectively. These sensing characteristics make PZTCC wafer suited to applications as a stress/strain sensor with stable linear responses.

Trace additions of strontium carbonate is effective in stabilizing the crystalline structure and retards volume shrinkage during the sintering of the PZT or PZTCC wafer. CSF and cement, as inorganic but fireproof admixture, lower the piezoelectric coefficient of PZTCC, whilst both equalizing the mechanical impedance factor of the PZT with concrete, and improving the fracture toughness of the corresponding PZTCC composite, where the ductile CSF achieved a fully netlike distribution. The overall results on piezoelectric sensitivity and toughness indicate a PZTCC wafer to be well suited as an intrinsic compatible sensor, which can be embedded in concrete structures for in situ monitoring.

\section{Conclusions}

(1) PZT nanoscale powder was successfully synthesized using the sol-gel process, from which a PZT wafer was created by sintering with trace of strontium carbonate producing fine and even cubic crystals within the 900-1100 nm range. CSF and cement were ground together with PZT nanoscale powder using ethanol as a dispersant to fabricate a PZTCC wafer in the same process as with the PZT wafer.

(2) CSF and cement incorporation weakens (110) perovskite crystallinity of PZTCC with cubic crystals, the $d_{33}$ reduces from $119.2 \mathrm{pC} / \mathrm{N}$ to $32.5 \mathrm{pC} / \mathrm{N}$, but narrows the hardness gap between the PZTCC and concrete, the corresponding microhardness is only $202.7 \mathrm{MPa}$, and improves intrinsic toughness and impedance-matching with common concrete. 
(3) A good linearity and fast response between the electrical signal against the loading of PZTCC wafer in either impulse or cyclic states is achieved with their corresponding sensitivity, linearity, and repeatability to $1.505 \mathrm{mV} / \mathrm{N}, 2.42 \%$, and $2.11 \%$, respectively.

The $d_{33}$ of this type of ductile piezoelectric PZTCC sensor is not high enough to enable in situ monitoring application. In the future, the corresponding piezoelectric property will be greatly improved by aging and temperature treatment method before developing as intrinsic sensor embedded in complex structures for real-time monitoring.

Author Contributions: J.L., L.L. and Q.L. conceived and designed the experiments; J.L., C.L. and C.Z. made the preliminary draft; J.L., K.L.C., B.W. and C.Z. made the revisions and discussions.

Acknowledgments: This work is supported by grants from the National Natural Science Foundation of China (Project No. 51878364, 51578297, 51678322 and 51650110509), Natural Science Foundation of Shandong Province (Project No. ZR2018MEE043 and 2017YFC0703603), and the National "111" project, and the Taishan Scholar Priority Discipline Talent Group program funded by the Shandong Province.

Conflicts of Interest: The authors declare that the research was conducted in the absence of any commercial or financial relationships that could be construed as a potential conflict of interest.

\section{Nomenclature}

PZT Lead-zirconate-titanate

XRD X-ray powder diffraction

SEM Scanning electronic microscopy

OP Optical microscope

CSF Chrysotile Fiber

SHM Structural health monitoring

PZTCC PZT/CSF/cement composite

$d_{33} \quad$ Piezoelectric coefficient

$e_{\mathrm{f}} \quad$ Repeatability

$\mathrm{U}_{\mathrm{F} . S} \quad$ Full-scale output of electricity (U)

$\Delta_{\max } \quad$ Maximum deviation between $\mathrm{U}$ - force (F) curve and the linear fitting line

$\Delta_{\max }^{\prime} \quad$ Maximum deviation in forward and reverse stroke of U-F curve

\section{References}

1. Han, B.; Yu, X.; Ou, J. Self-Sensing Concrete in Smart Structures; Butterworth-Heinemann, Elsevier Science: Amsterdam, The Netherlands, 2014.

2. Yi, J.H.; Kim, D.; Feng, M.D. Periodic seismic performance evaluation of highway bridges using structural health monitoring system. Struct. Eng. Mech. 2009, 31, 527-544. [CrossRef]

3. Li, X.X.; Ren, W.X.; Bi, K.M. FBG force-testing ring for bridge cable force monitoring and temperature compensation. Sens. Actuators A Phys. 2015, 223, 105-113. [CrossRef]

4. Ramakrishnan, M.; Rajan, G.; Semenova, Y.; Farrell, G. Overview of fiber optic sensor technologies for strain/temperature sensing applications in composite materials. Sensors 2016, 16, 99. [CrossRef] [PubMed]

5. Chen, P.W.; Chung, D.D.L. Carbon fiber reinforced concrete for smart structures capable of non-destructive flaw detection. Smart Mater. Struct. 1993, 2, 22-33. [CrossRef]

6. Wen, S.; Chung, D.D.L. Model of piezoresitivity in carbon fiber cement. Cem. Concr. Res. 2006, 36, $1879-1885$. [CrossRef]

7. Azhari, F.; Banthia, N. Cement-based sensors with carbon fibers and carbon nanotubes for peizoresistive sensing. Cem. Concr. Compos. 2012, 34, 866-873. [CrossRef]

8. Li, H.; Xiao, H.G.; Ou, J.P. Effect of compressive strain on electrical resistivity of carbon black-filled cement-based composites. Cem. Concr. Compos. 2006, 28, 824-828. [CrossRef]

9. Xiao, H.G.; Liu, M.; Wang, G.J. Anisotropic electrical and abrasion-sensing properties of cement-based composites containing aligned nickel powder. Cem. Concr. Compos. 2018, 87, 130-136. [CrossRef]

10. Han, B.G.; Han, B.Z.; Ou, J.P. Experimental study on use of nickel powder-filled Portland cement-based composite for fabrication of piezoresistive sensors with high sensitivity. Sens. Actuators A Phys. 2009, 149, 51-55. [CrossRef] 
11. Lan, C.M.; Xiao, H.G.; Liu, M.; Wang, G.J.; Ma, M.L. Improved piezoresistivity of cement-based composites filled with aligned nickel powder. Smart Mater. Struct. 2018, 27, 095003. [CrossRef]

12. Li, H.; Xiao, H.G.; Ou, J.P. A study on mechanical and pressure-sensitive properties of cement mortar with nanophase materials. Cem. Concr. Res. 2004, 34, 435-438. [CrossRef]

13. Ruan, Y.F.; Han, B.G.; Yu, X.; Zhang, W.; Wang, D.N. Carbon nanotubes reinforced reactive powder concrete. Compos. Part A Appl. Sci. Manuf. 2018, 112, 371-382. [CrossRef]

14. Schumacher, T.; Thostenson, E.T. Development of structural carbon nanotube-based sensing composites for concrete structures. J. Intell. Mater. Syst. Struct. 2014, 25, 1331-1339. [CrossRef]

15. Camacho-Ballesta, C.; Zornoza, E.; Garcés, P. Performance of cement-based sensors with CNT for strain sensing. Adv. Cem. Res. 2016, 28, 274-284. [CrossRef]

16. D'Alessandro, A.; Rallini, M.; Ubertini, F.; Materazzi, A.L.; Kenny, J.M. Investigations on scalable fabrication procedures for self-sensing carbon nanotube cement-matrix composites for SHM applications. Cem. Concr. Compos. 2016, 65, 200-213. [CrossRef]

17. Meoni, A.; D’Alessandro, A.; Downey, A.; García-Macías, E.; Rallini, M.; Materazzi, A.L.; Torre, L.; Laflamme, S.; Castro-Triguero, R.; Ubertini, F. An experimental study on static and dynamic strain sensitivity of embeddable smart concrete sensors doped with carbon nanotubes for SHM of large structures. Sensors 2018, 18, 831. [CrossRef] [PubMed]

18. Luo, J.L.; Chung, K.L.; Li, Q.Y.; Chen, S.J.; Li, L.; Hou, D.S.; Zhang, C.W. Piezoresistive properties of cement composites reinforced by functionalized carbon nanotubes using photo-assisted Fenton. Smart Mater. Struct. 2017, 26, 035025.

19. Konsta-Gdoutos, M.S.; Aza, C.A. Self-sensing carbon nanotube (CNT) and nanofiber (CNF) cementitious composites for real time damage assessment in smart structures. Cem. Concr. Compos. 2014, 53, 110-128. [CrossRef]

20. Galao, O.; Baeza, F.J.; Zornoza, E.; Garces, P. Strain and damage sensing properties on multifunctional cement composites with CNF admixture. Cem. Concr. Compos. 2014, 46, 90-98. [CrossRef]

21. Liu, Q.; Gao, R.D.; Tam, V.W.Y.; Li, W.G.; Xiao, J.Z. Strain monitoring for a bending concrete beam by using piezoresistive cement-based sensors. Constr. Bulid. Mater. 2018, 167, 338-347. [CrossRef]

22. Han, B.G.; Yu, X.; Ou, J.P. Effect of water content on the piezoresistivity of MWNT/cement composites. J. Mater. Sci. 2010, 45, 3714-3719. [CrossRef]

23. Kim, H.K.; Park, I.S.; Lee, H.K. Improved piezoresistive sensitivity and stability of CNT/cement mortar composites with low water-binder ratio. Compos. Struct. 2014, 116, 713-719. [CrossRef]

24. Proto, A.; Penhaker, M.; Bibbo, D.; Vala, D.; Conforto, S.; Schmid, M. Measurements of generated energy / electrical quantities from locomotion activities using piezoelectric wearable sensors for body motion energy harvesting. Sensors 2016, 16, 524. [CrossRef] [PubMed]

25. Kozielski, L.; Erhart, J.; Clemens, F.J. Light-intensity-induced characterization of elastic constants and d33 piezoelectric coefficient of PLZT single fiber based transducers. Sensors 2013, 13, 2419-2429. [CrossRef] [PubMed]

26. Hong, X.; Wang, H.; Wang, T.; Liu, G.; Li, Y.; Song, G.B. Dynamic cooperative identification based on synergetic for pipe structural health monitoring with piezoceramic transducers. Smart Mater. Struct. 2013, 22, 045003. [CrossRef]

27. Jaitanong, N.; Yimnirun, R.; Zeng, H.R.; Li, G.R.; Yin, Q.R.; Chaipanich, A. Piezoelectric properties of cement based/PVDF/PZT composites. Mater. Lett. 2014, 130, 146-149. [CrossRef]

28. Wang, D.; Song, H.; Zhu, H. Embedded 3D electromechanical impedance model for strength monitoring of concrete using a PZT transducer. Smart Mater. Struct. 2014, 23, 115019. [CrossRef]

29. Zhang, J.; Li, Y.; Du, G.F.; Song, G.B. Damage detection of L-shaped concrete filled steel tube (L-CFST) columns under cyclic loading using embedded piezoceramic transducers. Sensors 2018, 18, 2171. [CrossRef] [PubMed]

30. Li, Z.J.; Zhang, D.; Wu, K.R. Cement-based 0-3 piezoelectric composites. J. Am. Ceram. Soc. 2002, 85, 305-313. [CrossRef]

31. Dong, B.Q.; Li, Z.J. Cement-based piezoelectric ceramic smart composites. Compos. Sci. Technol. 2005, 65, 1363-1371. [CrossRef]

32. Li, Z.J.; Dong, B.Q.; Zhang, D. Influence of polarization on properties of 0-3 cement-based PZT composites. Cem. Concr. Compos. 2005, 27, 27-32. [CrossRef] 
33. Lan, K.H.; Chan, H.L.W. Piezoelectric cement-based 1-3 composites. Appl. Phys. A 2005, 81, 1451-1454.

34. Huang, S.F.; Chang, J.; Lu, L.C.; Chen, X. Preparation and polarization of 0-3 cement-based piezoelectric composites. Mater. Res. Bull. 2006, 41, 291-297. [CrossRef]

35. Chaipanich, A. Dielectric and piezoelectric properties of PZT-silica fume cement composites. Curr. Appl. Phys. 2007, 7, 532-536. [CrossRef]

36. Jaitanong, N.; Chaipanich, A.; Tunkasiri, T. Properties 0-3 PZT-Portland cement composites. Ceram. Int. 2008, 34, 793-795. [CrossRef]

37. Chaipanich, A.; Rianyoi, R.; Potong, R.; Jaitanong, N. Aging of 0-3 piezoelectric PZT ceramic-Portland cement composites. Ceram. Int. 2014, 40, 13579-13584. [CrossRef]

38. Li, Z.J.; Gong, H.Y.; Zhang, Y.J. Fabrication and piezoelectricity of 0-3 cement based composite with nano-PZT powder. Curr. Appl. Phys. 2009, 9, 588-591. [CrossRef]

39. Gong, H.Y.; Zhang, H.Y.; Che, S.W. Influence of carbon black on properties of PZT-cement piezoelectric composites. J. Compos. Mater. 2010, 44, 2547-2557.

40. Pan, H.H.; Chiang, C.K. Effect of aged binder on piezoelectric properties of cement-based piezoelectric composites. Acta Mech. 2014, 225, 1287-1299. [CrossRef]

41. Pan, H.H.; Lin, D.H.; Yang, R.H. High piezoelectric and dielectric properties of 0-3 PZT/cement composites by temperature treatment. Cem. Concr. Compos. 2016, 72, 1-8. [CrossRef]

42. Luo, J.L.; You, C.L.; Zhang, S.; Chung, K.L.; Li, Q.Y.; Hou, D.S.; Zhang, C.W. Numerical analysis and optimization on piezoelectric properties of 0-3 type piezoelectric cement-based materials with interdigitated electrodes. Appl. Sci. 2017, 7, 233. [CrossRef]

43. Zhang, S.Q.; Zhang, Y.M.; Li, Z.J. Ultrasonic monitoring of setting and hardening of slag blended cement under different curing temperatures by using embedded piezoelectric transducers. Constr. Bulid. Mater. 2018, 159, 553-560. [CrossRef]

44. Xu, K.; Deng, Q.S.; Cai, L.J.; Ho, S.C.; Song, G.B. Damage detection of a concrete column subject to blast loads using embedded piezoceramic transducers. Sensors 2018, 18, 1377. [CrossRef] [PubMed]

45. Xue, J.M.; Wan, D.M.; Lee, S.E.; Wang, J. Mechanochemical synthesis of lead zirconate titanate from mixed oxides. J. Am. Ceram. Soc. 1999, 82, 1687-1692. [CrossRef]

46. Brankovic, Z.; Brankovic, G.; Jovalekic, C.; Maniette, Y.; Cilense, M.; Varela, J.A. Mechanochemical synthesis of PZT powders. Mater. Sci. Eng. A 2003, 345, 243-248. [CrossRef]

47. Sharma, P.K.; Ounaies, Z.; Varadan, V.V.; Varadan, V.K. Dielectric and piezoelectric properties of microwave sintered PZT. Smart Mater. Struct. 2001, 10, 878-883. [CrossRef]

48. Deng, Y.; Liu, L.; Cheng, Y.; Nan, C.W.; Zhao, S.J. Hydrothermal synthesis and characterization of nanocrystalline PZT powders. Mater. Lett. 2003, 57, 1675-1678. [CrossRef]

49. Rao, K.R.M.; Rao, A.V.P.; Komarneni, S. Reactive PZT precursor powder by coprecipitation. Mater. Lett. 1996, 28, 463-467.

50. Barrow, D.A.; Petroff, T.E.; Tandon, R.P.; Sayer, M. Characterization of thick lead zirconate titanate films fabricated using a new sol gel based process. J. Appl. Phys. 1997, 81, 876-880. [CrossRef]

51. Mu, G.H.; Yang, S.Y.; Li, J.F.; Gu, M.Y. Synthesis of PZT nanocrystalline powder by a modified sol-gel process using water as primary solvent source. J. Mater. Process. Technol. 2007, 182, 382-386. [CrossRef]

52. Xu, Z.J.; Chu, R.Q.; Li, G.R.; Shao, X.; Yin, Q.R. Preparation of PZT powders and ceramics via a hybrid method of sol-gel and ultrasonic atomization. Mater. Sci. Eng. B 2005, 117, 113-118. [CrossRef]

53. Zheng, H.; Reaney, I.M.; Lee, W.E.; Jones, N.; Thomas, H. Effects of octahedral tilting on the piezoelectric properties of strontium/barium/niobium-doped soft lead zirconate titanate ceramics. J. Am. Ceram. Soc. 2002, 85, 2337-2344. [CrossRef]

54. Kusiorowski, R.; Zaremba, T.; Piotrowski, J.; Podworny, J. Utilisation of cement-asbestos wastes by thermal treatment and the potential possibility use of obtained product for the clinker bricks manufacture. J. Mater. Sci. 2015, 50, 6757-6767. [CrossRef]

55. Cross, W.M.; Sabnis, K.H.; Kjerengtroen, L.; Kellar, J.J. Microhardness testing of fiber-reinforced cement paste. ACI Mater. J. 2000, 97, 162-167.

(C) 2018 by the authors. Licensee MDPI, Basel, Switzerland. This article is an open access article distributed under the terms and conditions of the Creative Commons Attribution (CC BY) license (http:/ / creativecommons.org/licenses/by/4.0/). 